\title{
Three-dimensional Model of Maritime Search
}

\author{
Chun Zhang, Cheng Wang* \\ College of Mathematics and Physics, Huanggang Normal University, \\ Huanggang 438000, China \\ *Corresponding Author: wangc80@163.com
}

\begin{abstract}
Keywords: Maritime search and rescue; Differential equation; Global optimization; Region partition
\end{abstract}
\begin{abstract}
This paper establishes a three-dimensional maritime search and rescue plan by integrating a differential equation model, a global optimal model and an area partition algorithm. Based on the data of flight data and the loss time of communication for MAS MH370, we establish a differential equation model to determine the search scope of the sea area, and determine the place where the plane fall into water. After the plane falls into water, the plane will be moving in the water due to the effect of sustained ocean currents, thereby we establish a differential equation model. By solving this model, we obtain that the search scope is a circular area of 2000 square miles with a coordinate of circle center.
\end{abstract}

\section{Introduction}

With the development of economy, the plane has gradually become one of the convenient traffic. Plane flying does not be limited from the mountains, rivers, deserts, oceans and other geographical conditions, and can increase the number of plane according to the passenger source and the supply quantity. According to the statistics data from International Civil Aviation Organization, the average number of deaths in per one $\mathrm{km}$ is 0.04 people, and it is safer way than the train transportation. The plane's disadvantage is expensive, and heavily influenced by weather. Although the plane accident rate is very low, once the plane crash happens, very few people survived or even no survivors. Therefore, maritime search and rescue is a very complex and vital activity. When distress target location is unknown, we must carry out maritime search and rescue. Search operation is the most expensive, the most dangerous and the most complex part in the whole search and rescue process, is also the only way to find and rescue the survivors.

Making process of search action plan of the sea are generally considered more complex, so this paper takes a Boeing 737 crashed plane as an example, the searching process of sea action plan is divided into three steps: 1 ) Determine the specific scope of search area (i.e. to determine what kind of search power to participate in the action); 2) Determine the search partition and search way (i.e., to determine how these search powers act). And then we design a search plan after these three steps.

\section{The model}

In order to determine the specific scope of search area, the whereabouts process of plane is divided into two stages: (1) The falling process of the plane in the sky. In this stage, we will determine the place where fall into water. (2) The falling process of the plane in the water. Considering the sea buoyancy and the effect of ocean currents, we will determine the specific scope of search area.

Due to too many indexes for crash problem of different type plane, in order to simplify the problem, we take the Boeing 737 plane as an example. Suppose that the plane lost power at one point from $A$ to $B$ over the sea, the airplane flying in the air and generate the resistance $f$ with the opposite direction of its movement. Because of the Earth's gravity, the plane has the gravity $G$. And upper and lower wing surface flow velocity are inconsistent, so that the wing generates lift $F$. The projected point of the plane on the sea as the origin, northeast $45^{\circ}$ is the $X$-axis, and the vertical direction is $Y$-axis. In 
this coordinate system the initial coordinates of the plane is $(0,10000)$, and the initial plane velocity is parallel to the $X$-axis direction.

After plane lost power, speed and direction of movement, posture, air resistance and aerodynamic plane are changes over time change, so this is a typical problem of fluid dynamics. However, in order to simplify the model, we suppose that lift $F$ is a given value, and the direction is always perpendicular to the direction of motion of the plane.

Usually, when the object is not large and the moving speed is relatively low, we can suppose that the resistance is proportional to the speed if the object speed is less than $10 \mathrm{~m} / \mathrm{s}$, and in general within the range of considerable velocity and size of the object, it can be assumed that the resistance is proportional to the square of the speed from $10 \mathrm{~m} / \mathrm{s}$ to approach or exceed the speed of sound. If the object speed exceeds the speed of sound, then a sharp increase in resistance, and the resistance is proportional to high power of the speed [1].

The relative literature data are listed in the following Table 1.

Table 1 . Air density data under certain altitude

\begin{tabular}{lccccccc}
\hline Altitude (m) & 0 & 1000 & 2000 & 2500 & 3000 & 4000 & 5000 \\
\hline $\begin{array}{l}\text { Relative atmospheric } \\
\text { pressure }\end{array}$ & 1 & 0.881 & 0.774 & 0.724 & 0.677 & 0.591 & 0.514 \\
\hline \begin{tabular}{l} 
Relative air density \\
\hline $\begin{array}{l}\text { Absolute humidity } \\
(\mathrm{g} / \mathrm{m} 3)\end{array}$
\end{tabular} & 11 & 0.903 & 0.813 & 0.770 & 0.730 & 0.653 & 0.583 \\
\hline
\end{tabular}

Note: The atmospheric pressure $\mathrm{s}$ of standard state is 1 , and the relative air density is 1 . The air density around us is $1.293 \mathrm{~g} \cdot \mathrm{L}^{-1}$ at standard condition $(273 \mathrm{~K}, 101 \mathrm{KPa})$. According to the gas state equation, we can obtain the relationship between air density and elevation is

$$
\rho_{H}=\rho_{0}\left(1-\alpha H / T_{0}\right)^{4.26} \text {, }
$$

where $\rho_{H}$ is air density when the altitude is $H$; $\rho_{0}$ is the air density at standard state, and the air density under temperature conditions of zero centigrade is $1292 \mathrm{~g} / \mathrm{m}^{3} ; \alpha$ is the air temperature gradient which is about $0.0065 \mathrm{~K} / \mathrm{m} ; \mathrm{H}$ is the altitude $(\mathrm{m}) ; T_{0}$ is the absolute temperature, the value is $273 \mathrm{~K}$. By the relevant data are substituted into the formulas, we can get the relationship curve between air density and height as shown in Figure 1.

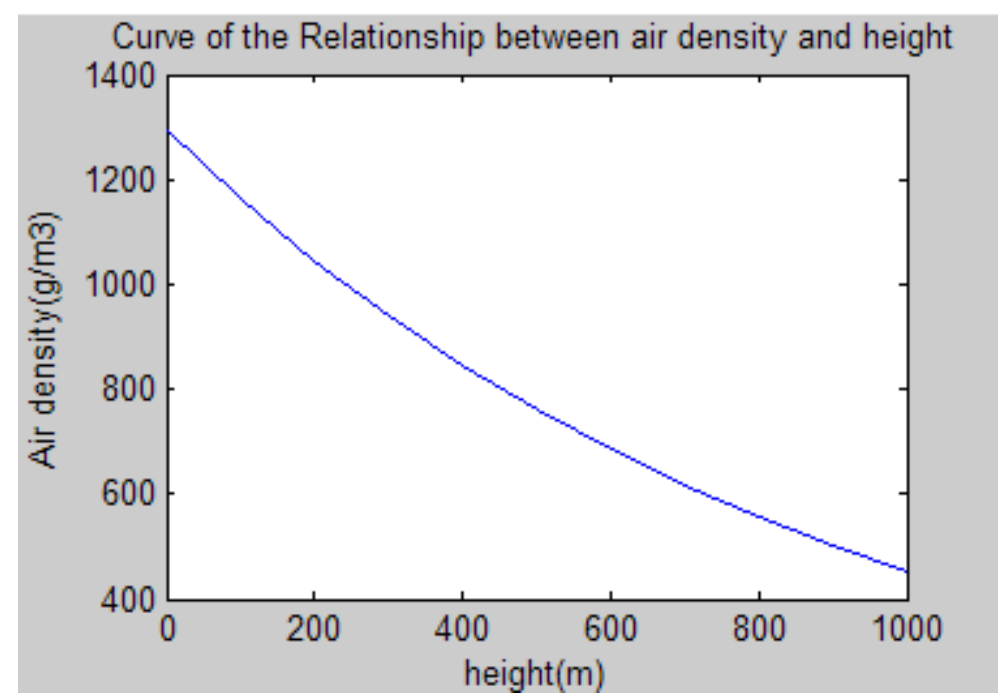

Figure 1. Relationship curve between air density and height

When the height is $10000 \mathrm{~m}$, we can obtain the air density is $574.68 \mathrm{~g} / \mathrm{m}^{3}$. Observing this curve, we can consider it as a linear relationship approximately. So we take the average value as the density of the atmosphere in this paper as follows. 


$$
\rho=\frac{1293+574.68}{2}=849 \mathrm{~g} / \mathrm{m}^{3}=0.849 \mathrm{~kg} / \mathrm{m}^{3} \text {. }
$$

According to the relative data of Boeing 737 plane, the weight of the plane is about $200000 \mathrm{~kg}$, windward area is about $130 \mathrm{~m}^{2}$, and the wind resistance coefficient of the plane is $C_{D}=0.08$.

$k=\frac{1}{2} \rho C_{D} S^{\prime}=4.418 \mathrm{~kg} / \mathrm{m}$.

Substitute $k$ into the initial conditions, we have

$$
\left.\frac{d x}{d t}\right|_{x=0}=222.2 \mathrm{~m} / \mathrm{s} ;\left.\frac{d y}{d t}\right|_{x=0}=0 ; y(0)=10000 ; x(0)=0 \text {. }
$$

By using the software of MATLAB, we can get the solutions are as follows.

$$
t=83.4 \mathrm{~s}, v_{x}=152.48 \mathrm{~m} / \mathrm{s}, v_{y}=135.46 \mathrm{~m} / \mathrm{s}
$$

and the falling trajectories of the plane is

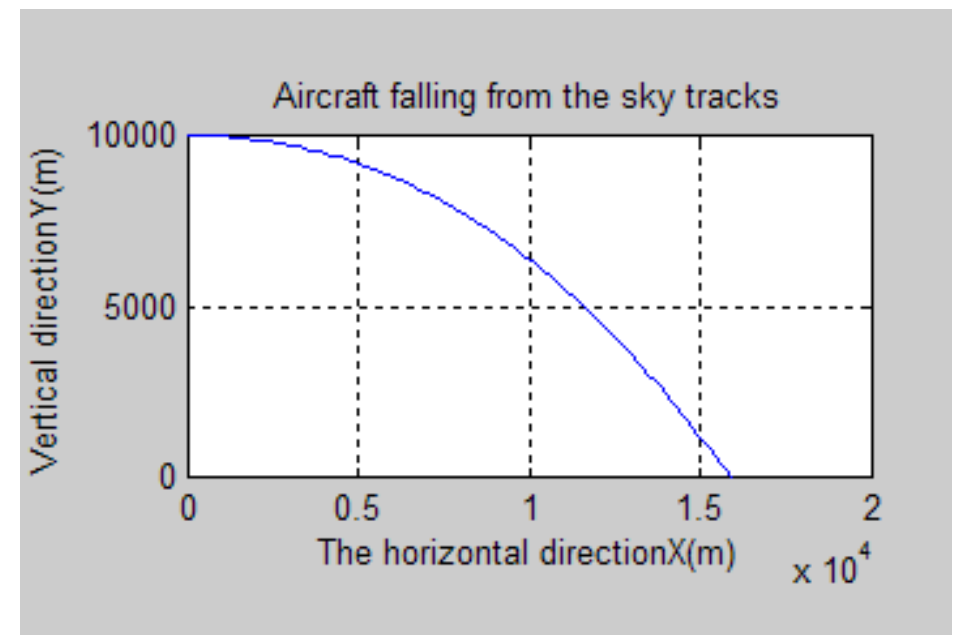

Figure 2. The falling trajectories of the plane

In Figure 2, the intersection point of parabola with $x$-axis is the place where the plane falls into the water. And its coordinate is $(15261,0)$, i.e., the plane crashed in the place of northeast $45^{\circ}$ and $15261 \mathrm{~m}$. And the location where the black box falls into water is (North latitude 7.1481 ${ }^{\circ}$, East longitude104.6571 ${ }^{\circ}$.

Because the stress has changed after the black box falls into the water, and black box will be influenced by ocean currents, this paper establishes the following model to describe the trajectory of black box, and then determine the specific scope of search area.

According to the known conditions, we can get

$$
k_{2}=\frac{1}{2} \rho C^{\prime} S^{\prime \prime \prime}=\frac{1}{2} \times 1.025 \times 10^{3} \times 0.12 \times 0.1=6.15
$$

where $C^{\prime}$ denotes the drag coefficient of sea water, here $C^{\prime}=0.12$ and $S^{\prime \prime \prime}$ shows the stress area of black box in seawater, here $S^{\prime \prime \prime}=0.1 \mathrm{~m}^{2}$.

As is well-known, $F=\rho \mathrm{gv}=100.45 \mathrm{~N}$, where $\rho=1.025 \times 10^{3} \mathrm{~kg} / \mathrm{m}^{3}$ is the density of seawater, $g=9.8 \mathrm{~N} / \mathrm{kg}$ represents the acceleration of gravity, and $v$ is the iron box volume. In general, $v=0.5 m \times 0.3 m \times 0.1 m$.

Consider the initial conditions

$\left\{\begin{array}{l}x^{\prime}(0)=v_{x} \\ y^{\prime}(0)=v_{y} \\ v_{z}=0.424\end{array}\right.$ and $\left\{\begin{array}{l}x(0)=0 \\ y(0)=0\end{array}\right.$

Tracking the black box which is affected by ocean currents, and getting the following Table 2 . 
Table 2. The black box of the settlement range

\begin{tabular}{ccc}
\hline $\mathrm{Y}$ axis & $\mathrm{X}$ axis & $\mathrm{Z}$ axis \\
\hline-1000 & $349.9474 \mathrm{~m}$ & 16.65 \\
-2000 & $881.3538 \mathrm{~m}$ & 43.94 \\
-3000 & $1280.868 \mathrm{~m}$ & 71.23 \\
\hline
\end{tabular}

This paper assumes that the black box falls into the sea water below $6000 \mathrm{~m}$, the horizontal distance of the black box operation in the sea is $46300 \mathrm{~m}$ by using differential equations. So this paper will search area which is roughly determined in 2000 square miles.

\section{Conclusions}

The paper selected differential equation of mechanics in the geometric model, and according to the given plane crash in the specific location, we find the relevant variables and equations related to the model, the use of aerodynamic formula and the second law of Newton formula help us obtain the drag coefficient of the relevant variable. Then decompose the trajectory and motion acceleration and make numerical differentiation respectively on the horizontal direction and the vertical direction. The sea three-dimensional search for global optimization problems in modeling, solving and result analysis. When sea to search for sea area is larger and the location of the distress target within the area, the need to carry out the comprehensive coverage of the area. When a large number of available search power available, how to choose the search for power can participate in the action to effectively cover the area is the practice of maritime search and urgently need to solve the problem. In this paper, the problem of abstract for the total with shipping and total plane constrained global optimization problem, sea three-dimensional search for global optimization model is established.

\section{Acknowledgments}

This work is supported by the Natural Science Foundation of Hubei Province (No. 2015CFB420), and the Reform Project of Transformation Development of Huanggang Normal University (No. xfg2015C19).

\section{References}

[1] S.W. Xing, Research on Global Optimization Model and Simulation of Joint Aeronautical and Maritime Search, Ph.D. Thesis, University of Dalian Maritime, 2012.

[2] S.A. Deloach, E.T. Matson, Y.H. Li, Exploiting agent oriented software engineering in cooperative robotics search and rescue, International Journal of Pattern Recognition and Artificial Intelligence 17 (2003) 817-835.

[3] C.J. Shi, K.Y. Xu, J. Peng, et al, Architecture of vision enhancement system for maritime search and rescue, In: Proceedings of 8th International Conference on ITS Telecommunications, Phuket, Thailand, Oct. 22-24, pp. 12-17, 2008.

[4] U.K. Izett, D. Unsalan, Integrating inertial and satellite navigation systems to improve performance for a maritime search and rescue aircraft, In: Proceedings of International Conference on Recent Advances in Space Technologies, pp. 610-615, 2003.

[5] Y. Liu, L. Ma, Q.Y. Xu, Solving multi-objective 0-1 programming by cellular ant algorithm, Systems Engineering 27 (2009) 119-122.

[6] J.H. Zhou. A study of evaluation methods for preferred rank and choice of SAR Ships, Navigation of China 62 (2005) 62-73. 